\title{
Material Storage Mechanism in Porous Nanocarbon - Comparison between Experiment and Simulation
}

\author{
Artur P. Terzyk ${ }^{1, *}$, Piotr A. Gauden ${ }^{1}$, Sylwester Furmaniak ${ }^{1}$, Piotr Kowalczyk ${ }^{2}$ \\ ${ }^{1} N$. Copernicus University, Department of Chemistry, \\ Physicochemistry of Carbon Materials Research Group, \\ Gagarin St. 7, 87-100 Toruń, Poland, \\ *e-mail: aterzyk@chem.uni.torun.pl \\ url: http://www.chem.uni.torun.pl/ aterzyk/ \\ ${ }^{2}$ Nanochemistry Research Institute, Department of Chemistry, \\ Curtin University of Technology, \\ P.O. Box U1987, Perth, 6845 Western Australia, Australia
}

(Received: 14 May 2012; revised: 19 June 2012; accepted: 22 June 2012; published online: 29 June 2012)

\begin{abstract}
We present first MD simulation results of $\mathrm{C}_{60}$ adsorption inside a single-walled carbon nanohorn. The assumed carbon nanohorn model and the values of the force field parameters lead to relatively good agreement between simulation and experiment. We show that the confinement of water and ethanol inside a carbon nanohorn strongly changes the properties of confined liquids leading to a decrease in the number of hydrogen bonds, and diffusion coefficients in comparison to bulk. The appearance of $\mathrm{C}_{60}$ inside the nanohorn leads to further decrease in diffusion coefficients of confined solvents.
\end{abstract}

Key words: fullerenes, $\mathrm{C}_{60}$, nanohorn, adsorption, molecular simulations

\section{INTRODUCTION}

Several years ago, Ajima et al. [1] studied the mechanism of materials storage in inner space of nanocarbons. They tried to visualize this process by adsorption of $\mathrm{C}_{60}$ in a single-walled carbon nanohorn (SWCNH). Studying the SWCNH filled with $\mathrm{C}_{60}$ with the High Resolution Transmission Electron Microscopy (HRTEM), the authors concluded that the mechanism of storage is not regular and fullerene molecules are heterogeneously distributed and mainly localised near the nanohorn tips. Therefore, fullerene molecules behave in a different way than it was observed for carbon nanotubes, where they were packed regularly. The authors also reported experimentally determined distribution of $\mathrm{C}_{60}$ inside a carbon nanohorn by plotting the graph showing the frequency of fullerene molecules with respect to the local curvature of graphite walls defined by the local diameter of a nanohorn.

The results reported by Ajima et al. [1] are important not only for fundamental studies of the storage mechanism.
Recent results published by Miyako et al. [2] demonstrated that $\mathrm{C}_{60}$ molecules encapsulated in SWCNH can be released by NIR laser irradiation, suggesting that SWCNHs are potentially useful for a photocontrolled release of drugs. Therefore, the knowledge of the influence of confinement (as well as solvent type) on the structure of adsorbed $\mathrm{C}_{60}$ seems to be very important.

The major purpose of this study is, by using Molecular Dynamics (MD) simulation, to identify the mechanism of $\mathrm{C}_{60}$ adsorption inside SWCNH. Moreover, we tried to identify the structure of solvent inside a nanohorn, as well as the influence of adsorbed $\mathrm{C}_{60}$ on the structure of solvent. We expect to obtain a similarity between determined from simulation, and experimentally observed distribution curves (see Fig. 4 in [1]) of fullerenes inside SWCNH. However, in this case one should remember that since we use in our simulation only a single nanohorn as the representation of the whole dahlia-flower structure, it is practically impossible to obtain perfect agreement between simulation and experiment. Moreover, careful observation of HRTEM pictures revealed that real carbon nanohorns 
possess distributed diameters and have different lengths [1]. Also the shape of a nanohorn is not perfect in some cases, and one can observe some highly deformed cones even having ink-bottled structures.

In our simulation by replacing ethanol with water we also study the influence of solvent on the distribution of fullerenes inside a nanohorn, since Ajima et al. [1] adsorbed $\mathrm{C}_{60}$ only from ethanol solution.

\section{MOLECULAR DYNAMICS SIMULATION}

To study the configuration of $\mathrm{C}_{60}$ in a nanohorn at $298 \mathrm{~K}$ we applied the GROMACS simulation package [3], and the OPLSAA force field described in detail previously [4-11]. The topology of a water molecule can be represented in this package as the TIP4P model, applied in the current study. We used the TIP4P model because simple empirical three-point-charge water models such as TIP3P, SPC/E, and TIP4P are quite successful in describing the properties of water [12]. It is only in the solid phase where we find that the performance of these models is different in a significant way, but the solidification of water has not been expected in the current study. The simplicity of the TIP4P functions is also attractive from a computational standpoint [13]. We also used the TIP4P model since it is most popular in simulations of water/nanotube systems [14].

The force field parameters for an ethanol molecule were exactly the same as studied by Taylor and Shields - see Table 1 in [15]. The force field parameters for $\mathrm{C}_{60}$ fullerene simulation were taken from the study of Malaspina et al. [16] who simulated the behaviour of $\mathrm{C}_{60}$ in ethanol solution.

In our simulation we studied 4, 8, 12 and 16 fullerene molecules inside an idealized carbon nanohorn model. This nanohorn was generated by cutting wedge (the disclination angle $300^{\circ}$ ) from the graphene sheet and stitching its edges (see Fig. 2 in [17]). The final structure contains 3002 carbon atoms. The opening angle was assumed as equal to $19.2^{\circ}$ - this angle is very close to $20^{\circ}$ the most often experimentally observed for SWCNH [17, 18]. To speed up the simulation we applied the following procedure. We introduced a fixed number $\left(4,8,12\right.$ and 16) of $\mathrm{C}_{60}$ molecules inside the empty carbon nanohorn and next, the energy of this system was minimized using the Monte Carlo (MC) simulation in the Canonical Ensemble. During the MC we assumed the same values of carbon - carbon potential parameters as during the MD simulation. Next, after equilibration the solvent was introduced (ca. 24000 of water and 8000 of ethanol molecules) and the MD simulation was performed using the Berendsen barostat $(1 \mathrm{~atm})$ and thermostat $(298 \mathrm{~K})$. The equilibration time of MD simulation was at least $5 \mathrm{~ns}$.

\section{RESULTS AND DISCUSSION}

First of all, to check our fullerene model we tried to recover the results published by Malaspina et al. [16]. The comparative results of diameter oscillation for isolated and solvated in ethanol fullerene $\mathrm{C}_{60}$ molecule during $\mathrm{MD}$ simulations, and the comparison of the Radial Distribution Functions (RDFs) calculated in [16] and during our simulation shows very good agreement between results. Figure 1 shows applied notation, the details of simulation boxes, and the snapshots from equilibrated systems (note that some figures were created using the VMD program [19, 20]).
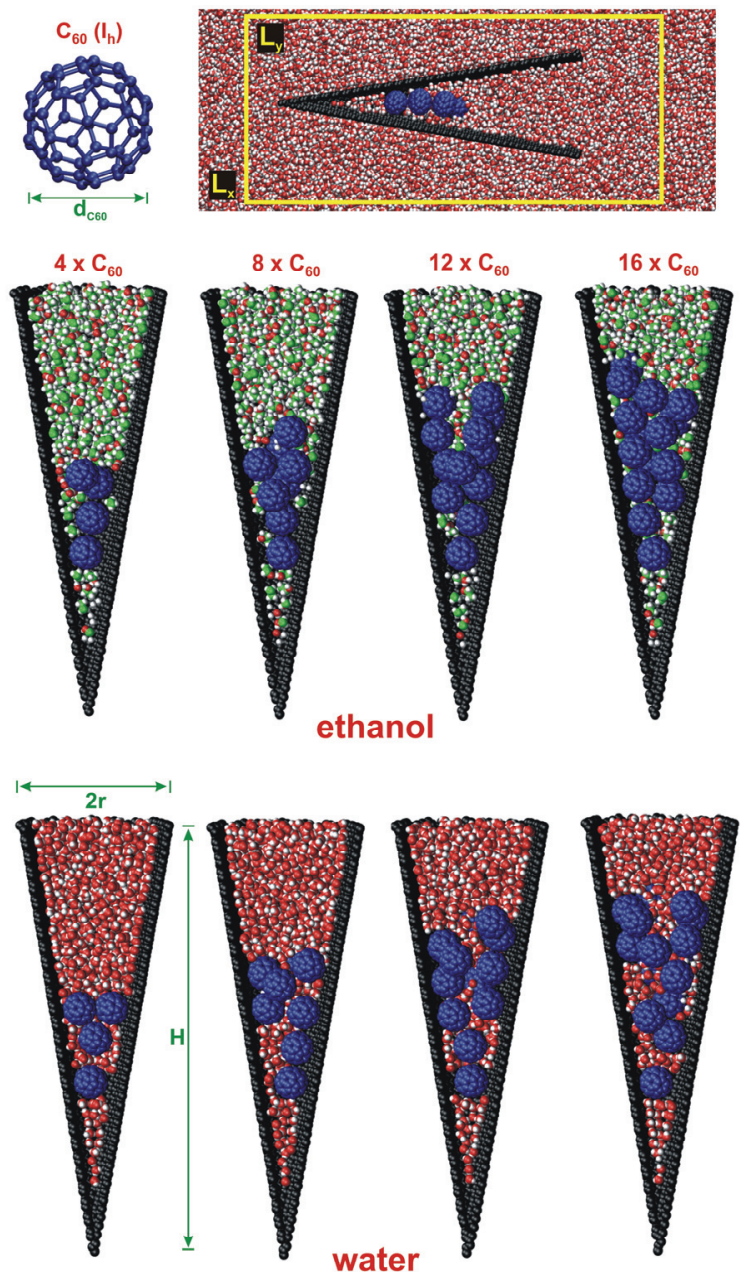

Fig. 1. The schematic representation of simulation box, applied methodology and the equilibrium configurations of fullerene molecules in water and in ethanol solutions 
The comparison between simulation and experimental data published by Ajima et al. [1] is shown in Fig. 2a for both studied solvents as well as for a different number of $\mathrm{C}_{60}$ molecules. As one can see, for a relatively small number of fullerene molecules introduced into simulation box we observe quite good agreement between distributions obtained from the experiment and determined from simulations. There is no remarkable difference between distribution of $\mathrm{C}_{60}$ in water and in ethanol. Therefore, simulation results show that due to large energy of intermolecular interactions between fullerenes and walls we do not expect that the replacement of ethanol with water will change the structure of fullerenes deposited inside SWCNH.

In Fig. $2 b$ we show the density profiles. During the calculations it was assumed that the centre of a water molecule is located on an oxygen atom, and the centre of ethanol is at the centre of mass of this molecule. One can observe that in fact $\mathrm{C}_{60}$ molecules are located at the tip ends and at the vicinity of the nanohorn walls. Moreover, we also observe high density of both solvents at the walls, as well as the layered structure, especially pronounced for the case of water.
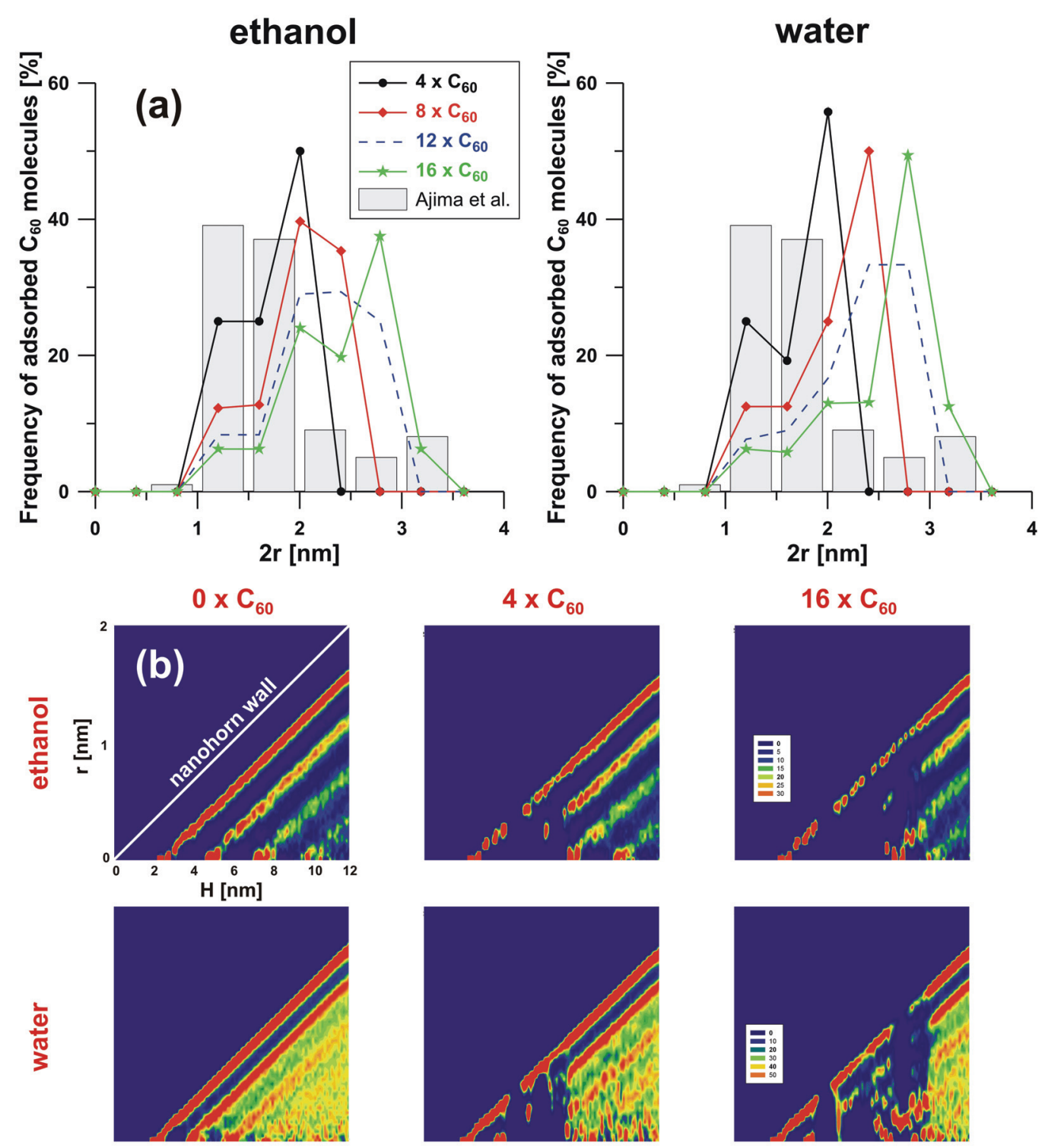

Fig. 2. (a) Frequency of adsorbed $\mathrm{C}_{60}$ molecules vs. nanohorn curvature radius - the comparison between simulation (lines) and experimental data published by Ajima et al. [1] (bars). (b) The density (in molecules $/ \mathrm{nm}^{3}$ ) profiles (see Fig. 1) - one can observe the layered structure of solvent and the location of $\mathrm{C}_{60}$ at the vicinity of carbon nanohorn walls 


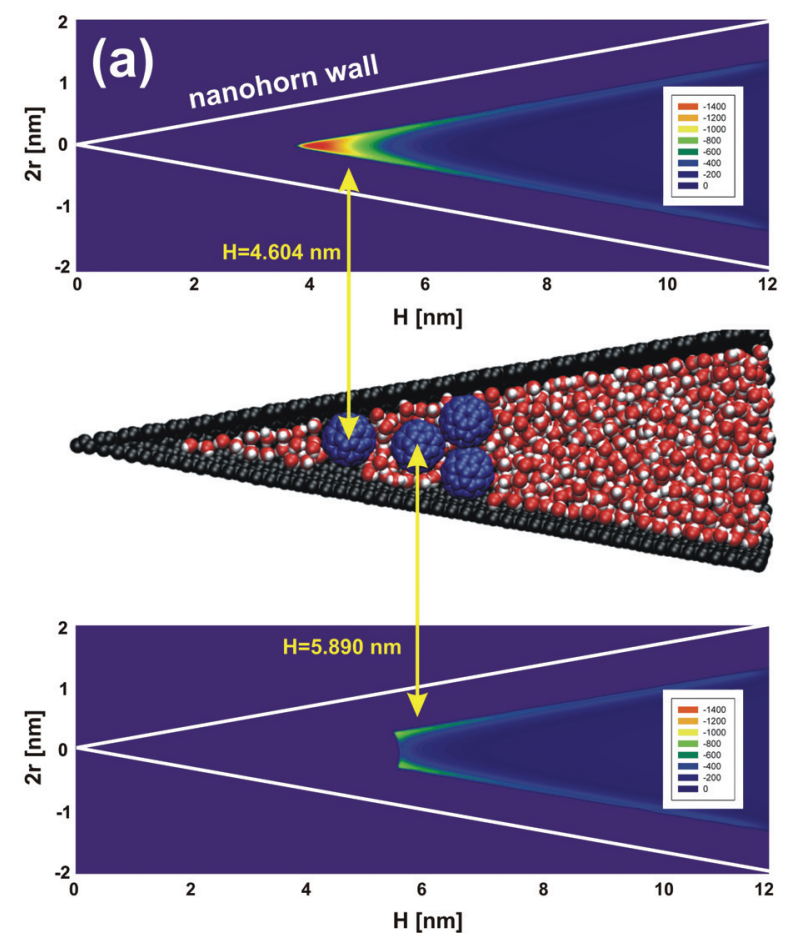

(b)

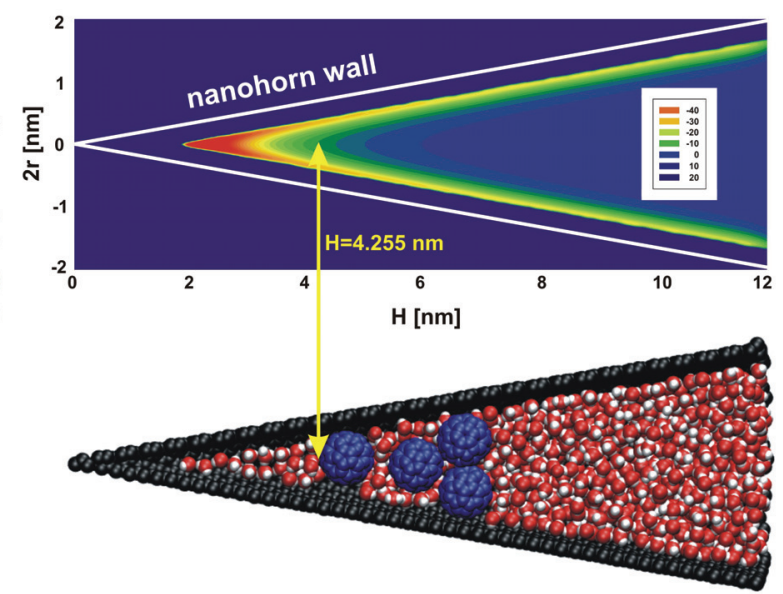

Fig. 3. (a) The profile of Lennard-Jones solid-fluid ( $s f$ ) (reduced by $\varepsilon_{s f}$ ) potential energy of interaction between $\mathrm{C}_{60}$ and nanohorn $(2 r-H$ plane). The points represent the fullerene centre shifted in the axis plane. Bottom figure shows the potential after adsorption of the first fullerene (at the tip). (b) The profile of Lennard-Jones solid-fluid ( $s f$ ) (reduced by $\varepsilon_{s f}$ ) potential energy of interaction for $\mathrm{O}$ atom in the $2 r-H$ plane

The data shown in Fig. 3a provide the justification of the $\mathrm{C}_{60}$ location at the nanohorn walls (for a larger number of adsorbed fullerenes), and explains the experimental conclusion about the preferred localisation of fullerene near the nanohorn tips (for a small number of fullerenes inside nanohorns). This figure shows the potential energy inside SWCNH. This reduced potential energy was calculated introducing $\mathrm{C}_{60}$ inside our nanohorn model. As one can observe, the location of only one $\mathrm{C}_{60}$ molecule (located at the tip) is influenced by the enhancement of potential energy caused by overlapping interaction energy from the surrounding carbon atoms of the nanohorn wall (to show this effect more clearly we plotted on the bottom figure the potential profile calculated after adsorption of the first fullerene at the tip).

A similar potential energy profile plot is shown in Fig. $3 b$ but for an oxygen atom of water (it should be noted that negligibly small qualitative differences were recorded between the profiles obtained for a carbon atom of the ethanol molecule and the oxygen atom mentioned above).

The data collected in this figure show that at the tip end molecules are in an overlapping potential field. Thus this field causes the creation of a unique phase in a conical potential confinement.
Figure 4 shows the average number of molecules in slices (thickness ca. $0.3 \mathrm{~nm}$ ) as well as the average number of created hydrogen bonds calculated using the procedure proposed by Gordillo and Marti [21]. As one can see, with a rise in the number of adsorbed fullerenes inside the carbon nanohorn the number of water molecules, and subsequently the number of formed hydrogen bonds decreases. The number of hydrogen bonds calculated per a single water molecule in the bulk at $298 \mathrm{~K}$ is equal to 3.4. Saiz et al., who used the OPLSAA force field and MD simulations of the bulk ethanol molecules, concluded that the average number of ethanol hydrogen bonds per single molecule is (at $T=298 \mathrm{~K}$ ) equal to 1.88 [22]. One can observe that those values are obtained for bulky solvents, i.e. at large distances from the nanohorn tip (see Fig. 4).

Very interesting is the structure of water and ethanol at the tip end. For molecules closed in the potential field shown in Fig. 3 - between a $\mathrm{C}_{60}$ fullerene and the tip - we observe strong reduction in the number of hydrogen bonds formed per single molecule in comparison to the bulk. In this nanoconfinement we observe the creation of conical water and ethanol structures and, as it can be seen from Fig. 4, the presence of fullerene has a larger influence on the number of hydrogen bonds created between ethanol 

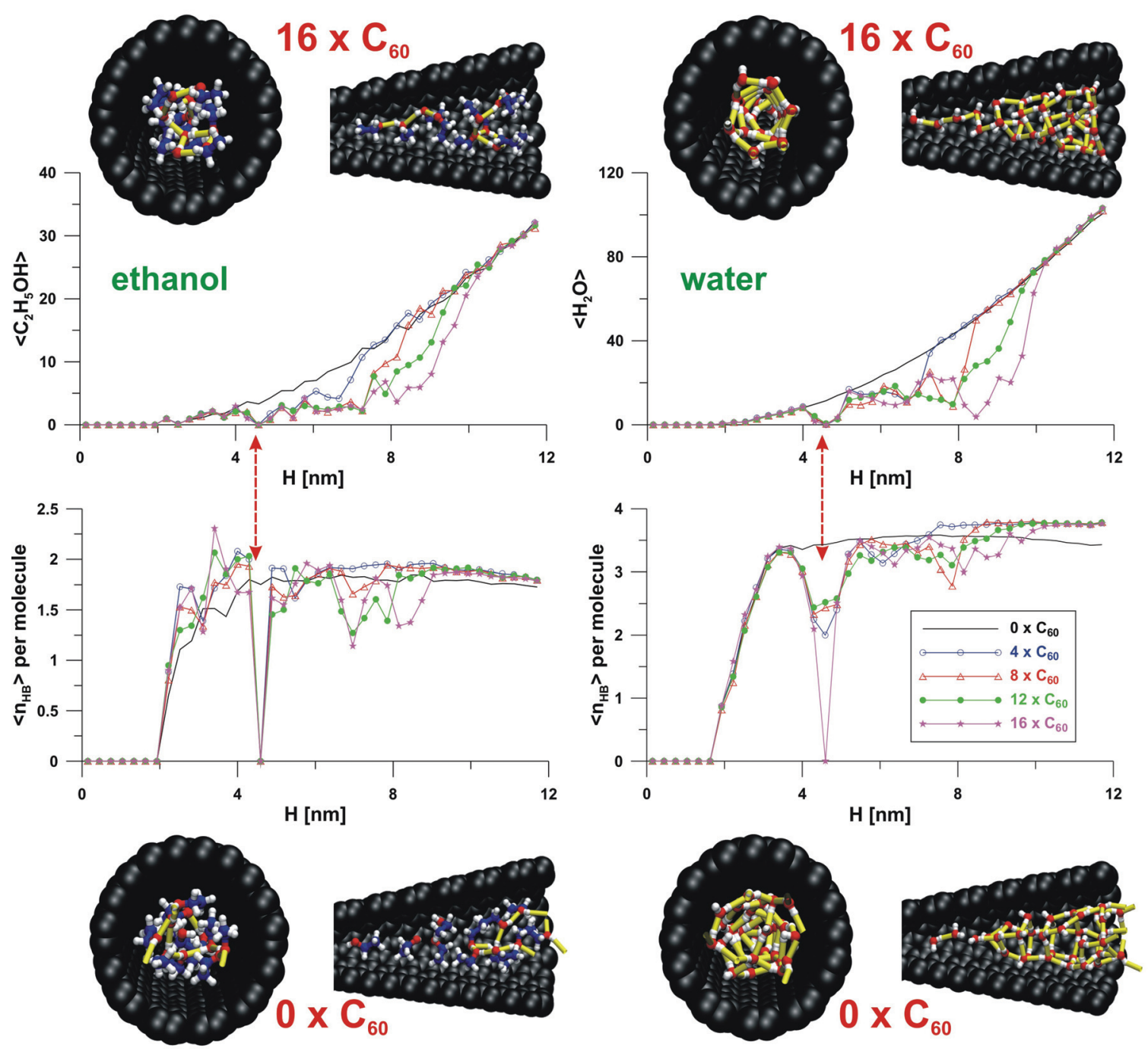

Fig. 4. The number of solvent molecules and hydrogen bonds created between them (marked in yellow) in a empty nanohorn filled with $16 \times \mathrm{C}_{60}$ molecules (up to $H=2.555 \mathrm{~nm}$ (see red arrows and Fig. 3))

Table 1. Self diffusion coefficients of solvents caluclated for bulk and under confinement

\begin{tabular}{c|c|c}
\hline System & Water $\left(\mathrm{D} 10^{5} \mathrm{~cm}^{2} / \mathrm{s}\right)$ & Ethanol $\left(\mathrm{D} 10^{5} \mathrm{~cm}^{2} / \mathrm{s}\right)$ \\
\hline bulk & & $2.1504(+/-0.0037)$ \\
\hline $0 \times \mathrm{C}_{60}$ & $2.2234(+/-0.0943)$ & $1.8006(+/-0.0342)$ \\
\hline $4 \times \mathrm{C}_{60}$ & $2.1632(+/-0.0217)$ & $1.1804(+/-0.0142)$ \\
\hline $8 \times \mathrm{C}_{60}$ & $2.0361(+/-0.0634)$ & $0.8058(+/-0.0573)$ \\
\hline $12 \times \mathrm{C}_{60}$ & $2.0067(+/-0.0275)$ & $0.6797(+/-0.0250)$ \\
\hline $16 \times \mathrm{C}_{60}$ & $1.9312(+/-0.0916)$ & $0.6117(+/-0.0910)$ \\
\hline
\end{tabular}

molecules than between water ones (ethanol molecule has a larger collision diameter). The structure of conical water and ethanol is shown in Fig. 4. Table 1 summarizes the self diffusion coefficients obtained in this analysis. They were calculated from the period of 500 ps for molecules inside nanohorn. It can be seen that the presence of fullerenes in a small amount changes the diffusion coefficient of water. Contrary for ethanol the drastic changes are observed. In both cases we observe a progressive decrease in the diffusion coefficient value with the rise in the number of fullerenes inside.

\section{CONCLUSIONS}

The major conclusions of our study are as follows. The assumed carbon nanohorn model and the values of the 
force filed parameters lead to relatively good agreement between simulation and experiment. The distribution of $\mathrm{C}_{60}$ inside $\mathrm{SWCNH}$ is similar to observed experimentally; moreover, this distribution is almost the same in water as in ethanol. The calculation of the enhancement of potential energy due to conical symmetry shows that it is exerted only on the first $\mathrm{C}_{60}$ molecule. This is in agreement with experiment and simulation showing that the molecules are mainly adsorbed at the tip ends. However, all solvent molecules closed in the space between tip and first adsorbed fullerene are in this potential field. This causes the creation of a unique phase with a reduced (water) or slightly larger (ethanol) number of hydrogen bonds, comparing to the bulk. The number of hydrogen bonds formed by water varies between 1 and 3 (for bulk water we observe 3.4) and for ethanol it changes in the range 1-2 (i.e. at the vicinity of $\mathrm{C}_{60}$ it is slightly larger than in the bulk). This suggests that conical hydrogen bonded liquids reveal different and interesting properties in comparison to the bulk, for example the number of hydrogen bonds and/or self diffusion coefficients. Additional properties of confined solvents will be studied in the future.

\section{Acknowledgments}

Authors acknowledge a computational grant from the Poznan Supercomputing and Networking Center (PCSS).

\section{References}

[1] K. Ajima, M. Yudasaka, K. Suenaga, D. Kasuya, T. Azami, S. Iijima, Material storage mechanism in porous nanocarbon. Adv. Mater. 16, 397-401 (2004).

[2] E. Miyako, H. Nagata, K. Hirano, Y. Makita, T. Hirotsu, Photodynamic release of fullerenes from within carbon nanohorn. Chem. Phys. Lett. 456, 220-222 (2008).

[3] E. Lindahl, B. Hess, D. van der Spoel, GROMACS 3.0: A package for molecular simulation and trajectory analysis. J. Mol. Model. 7, 306-317 (2001).

[4] A.P. Terzyk, P.A. Gauden, S. Furmaniak, R.P. Wesołowski, P.J.F. Harris, P. Kowalczyk, Adsorption from aqueous solutions on opened carbon nanotubes - organic compounds speed up delivery of water from inside. Phys. Chem. Chem. Phys. 11, 9341-9345 (2009).

[5] A.P. Terzyk, A. Pacholczyk, M. Wiśniewski, P.A. Gauden, Enhanced adsorption of paracetamol on closed carbon nanotubes by formation of nanoaggregates: Carbon nanotubes as potential materials in hot-melt drug deposition-experiment and simulation. J. Colloid Interface Sci. 376, 209-216 (2012).
[6] A.P. Terzyk, P.A. Gauden, S. Furmaniak, R.P. Wesołowski, P. Kowalczyk, Activated carbon immersed in waterthe origin of linear correlation between enthalpy of immersion and oxygen content studied by molecular dynamics simulation. Phys. Chem. Chem. Phys. 12, 10701-10713 (2010).

[7] A.P. Terzyk, S. Furmaniak, P.A. Gauden, P.J.F. Harris, R.P. Wesołowski, P. Kowalczyk, Virtual porous carbon (VPC) models - application in study of fundamental activated carbon properties by molecular simulations. in: J.F. Kwiatkowski (ed.) Activated Carbon: Classification, Properties and Applications. Nova Science Publishers, New York, p. 355-376, 2011.

[8] R.P. Wesołowski, S. Furmaniak, A.P. Terzyk, P.A. Gauden, Simulating the effect of carbon nanotube curvature on adsorption of polycyclic aromatic hydrocarbons. Adsorption 17, 1-4 (2011).

[9] P.A. Gauden, A.P. Terzyk, R. Pieńkowski, S. Furmaniak, R.P. Wesołowski, P. Kowalczyk, Molecular dynamics of zigzag single walled carbon nanotubes immersion in water. Phys. Chem. Chem. Phys. 13, 5621-5629 (2011).

[10] R.P. Wesołowski, A.P. Terzyk, Pillared graphene as a gas separation membrane. Phys. Chem. Chem. Phys. 13, 17027-17029 (2011).

[11] A.P. Terzyk, P.A. Gauden, W. Zieliński, S. Furmaniak, R.P. Wesołowski, K.K. Klimek, First molecular dynamics simulation insight into the mechanism of organics adsorption from aqueous solutions on microporous carbons. Chem. Phys. Lett. 515, 102-108 (2011).

[12] J.L.F. Abascal, C. Vega, The water forcefield: Importance of dipolar and quadrupolar interactions. J. Phys. Chem. C 111, 15811-15822 (2007).

[13] W.L. Jorgensen, J. Chandrasekhar, J.D. Madura, R.W. Impey, M.L. Klein, Comparison of simple potential functions for simulating liquid water. J. Chem. Phys. 79, 926-935 (1983).

[14] A. Alexiadis, S. Kassinos, Molecular simulation of water in carbon nanotubes. Chem. Rev. 108, 5014-5034 (1008).

[15] R.S. Taylor, R.L. Shields, Molecular-dynamics simulations of the ethanol liquid-vapor interface. J. Chem. Phys. 119, 12569-12576 (2003).

[16] T. Malaspina, E.E. Fileti, R. Rivelino, Structure and $U V$ Vis spectrum of $C_{60}$ fullerene in ethanol: A sequential molecular dynamics/quantum mechanics study. J. Phys. Chem. B 111, 11935-11939 (2007).

[17] M. Yudasaka, S. Iijima, V.H. Crespi, Single-wall carbon nanohorns and nanocones. Topics in Applied Physics 111, 605-629 (2008).

[18] Y. Tao, D. Noguchi, Ch.-M. Yang, H. Kanoh, H. Tanaka, M. Yudasaka, S. Iijima, K. Kaneko, Conductive and mesoporous single-wall carbon nanohorn/organic aerogel composites. Langmuir 23, 9155-2157 (2007).

[19] W. Humphrey, A. Dalke, K. Schulten, VMD - visual molecular dynamics. J. Mol. Graphics 14, 33-38 (1996).

[20] http://www.ks.uiuc.edu/Research/vmd/.

[21] M.C. Gordillo, J. Marti, Hydrogen bond structure of liquid water confined in nanotubes. Chem. Phys. Lett. 329, 341345 (2000)

[22] L. Saiz, J.A. Padro, E. Guardia, Dynamics and hydrogen bonding in liquid ethanol. Mol. Phys. 97, 897-905 (1999). 


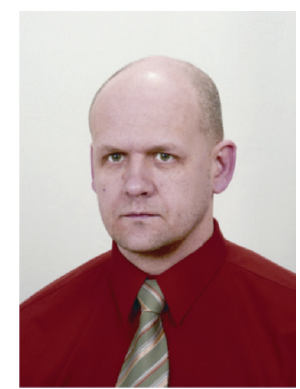

ARTUR P. TerzYK. Personal data: Born on November 22, 1967, in Żnin, Poland. Education: 1991: M.Sc. in Physical Chemistry from Nicolaus Copernicus University in Toruń; 1995: Ph.D. in Physical Chemistry from Nicolaus Copernicus University in Torun, Thesis: "The Study on the Molecular Interactions in the System: Adsorbate - Microporous Activated Carbon"; 2005: Habilitation (awarded by Prime Minister of Poland) in Physical Chemistry from Nicolaus Copernicus University in Torun, Thesis: "Acivated Carbon - New Methods of Characterization and the Application in Adsorption of Biologically Active Substances". Professional Experience: One of the founders and members of "Physicochemistry of Carbon Materials Research Group" at Department of Chemistry Nicolaus Copernicus University, Torun since October 1991 (head of the group since October 2009); invited speaker at Carbon Based Nanoporous Materials for Future Challenge (CBNM) conference (Japan, Chiba University, 2008); keynote speaker at Carbon conference in Biarritz, France (2009); invited speaker at the workshop before Carbon 2012 conference (Kraków); keynote speaker at Carbon 2012 conference, member of the scientific committee of the International Symposium on Surface Heterogeneity in Adsorption and Catalysys (ISSHAC) (2009 and 2012). Scientific Activities Honours: In 1995 the Scholarship of Foundation for Polish Science. Publications: 2 monographs: "The Theory of Adsorption of Gases and Vapours in Micropores of Carbonaceous Materials" and "Carbon Materials - Theory and Practice"; original papers: more than 170 (excluding conference materials); the total number of citations of all previous publications: 1020; index: H: 15 (excluding self citations, Scopus, 30.04.2012). Current research interests: adsorption from gaseous phase; adsorption of biologically active compounds from aqueous solutions on to carbons; thermodynamics of adsorption; adsorption calorimetry; and theory of adsorption.

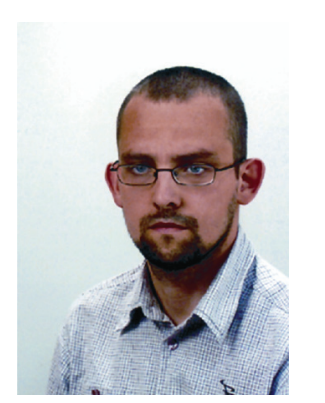

Piotr A. GAUden. Personal data: Born on January 2, 1973, in Lidzbark Welski, Poland. Education: 1997: M.Sc. in Physical Chemistry from Nicolaus Copernicus University in Toruń; 2001: Ph. D. in Physical Chemistry from Nicolaus Copernicus University in Torun, Thesis: "Theoretical Description of the Structural and Energetic Heterogeneity of Carbonaceous Materials". Professional Experience: One of the founders and members of "Physicochemistry of Carbon Materials Research Group" at Department of Chemistry, Nicolaus Copernicus University, Torun since October 1997. Scientific Activities Honours: In 2000, the Scholarship of Foundation for Polish Science. Publications: 2 monographs: "The Theory of Adsorption of Gases and Vapours in Micropores of Carbonaceous Materials" and "Carbon Materials - Theory and Practice"; original papers: more than 130 (excluding conference materials); the total number of citations of all previous publications: 505; index: H: 11 (excluding self citations, Scopus, 30.04.2012). Current research interests: physical chemistry; adsorption; thermodynamics of adsorption; numerical recipes; and computer simulations (GCMC and MD).

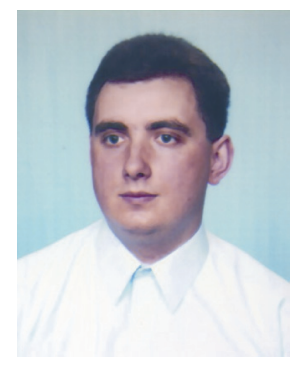

SylWester FurmaniaK. Personal data: Born on December 28, 1979, in Złotów, Poland. Education: 2005: M.Sc. in Physical Chemistry from Nicolaus Copernicus University in Torun. 2010: Ph.D. in Physical Chemistry from Nicolaus Copernicus University in Torun, Thesis: "Sorption Equilibria in Pores of Carbonaceous Materials Analytical Models and Computer Simulations". Professional Experience: member of "Physicochemistry of Carbon Materials Research Group" at Department of Chemistry, Nicolaus Copernicus University, Torun since October 2005. Scientific Activities Honours: In 2009 and 2010, the Scholarship of Foundation for Polish Science. Publications: Original papers: more than 60 (excluding conference materials); the total number of citations of all previous publications: 131; index: H: 7 (excluding self citations, Scopus, 30.04.2012). Current research interests: modeling of adsorption and related energetic (using Monte Carlo, molecular dynamics simulations and analytical models).

Piotr Kowalczyk. Personal data: Born on October 22, 1975, in Poniatowa, Poland. Education: 1999: M.Sc. in Chemistry from the Military University of Technology; 2001: Postgraduate Computer Science Studies "Software engineering" from the Jagiellonian University; 2004: $\mathrm{PhD}$ degrees in Chemistry from the Chiba University. Professional Experience: Since 1999, close cooperation with "Physicochemistry of Carbon Materials Research Group" from N. Copernicus University, Torun, Poland; past member of: "Kaneko Laboratory" at the Department of Chemistry, Chiba University, Chiba, Japan; "Soft Condensed Research Group" at the Institute of Physical Chemistry, Polish Academy of Sciences, Warszawa, Poland; "Adsorption and Reaction in Nanoporous Materials Research Group" at the School of Chemical Engineering, University of Queensland, Brisbane, Australia; "Materials Modeling and Simulation Group" at Applied Physics, Royal Melbourne Institute of Technology, Melbourne, Australia; current member: "Theoretical and Computational Research Group" at the Nanochemistry Research Institute, Department of Chemistry, Curtin University of Technology, Pert, Australia. Scientific Activities Honours: 2006: Postdoctoral Research Fellowship, The University of Queensland; 2010: Senior Research Fellowship, Curtin University of Technology. Publications and Citations: 1 monograph: "Carbon Materials - Theory and Practice"; original papers: more than 85 (excluding conference materials); the total number of citations of all previous publications: 519; index: H: 12 (excluding self citations, Scopus, 30.04.2012). Current research interests: theoretical and computational chemistry; mathematical physics; chromatography; and adsorption. 